\title{
Correlation between Left Atrial Appendage and Right Atrial Appendage Velocities in Different Pathologies and Normal Subjects
}

\author{
Mohaddeseh Behjati ${ }^{1}$, Saman Rostambeigi (iD) ${ }^{1}$ and Azin Alizadehasl (iD) ${ }^{1, *}$ \\ ${ }^{1}$ Rajaie Cardiovascular Medical and Research Center, Tehran, Iran \\ "Corresponding author: Oncology Department and Research Center, Rajaie Cardiovascular Medical and Research Center, Tehran, Iran. Tel: +98-2123922190, Email: \\ alizadeasl@gmail.com
}

Received 2020 July 13; Revised 2020 July 19; Accepted 2020 July 21.

Keywords: Left Atrial Appendage, Right Atrial Appendage, Velocity, Atrial Dysfunction, Correlation

\section{Dear Editor,}

Anatomy and function of left atrial appendage (LAA) are well characterized in healthy and disease states but little attention has yet been paid to right atrial appendage (RAA). The correlation between LAA and RAA has been observed in a small condition as atrial fibrillation, but their correlation in other conditions has not totally been evaluated. In this investigation, we aimed to evaluate the correlation between LAA and RAA velocities in different states. In this study, we have evaluated LAA and RAA velocities in echocardiographic 2D and Doppler study in 40 patients with different pathologies such as valvular and non-valvular atrial fibrillation, mitral stenosis, cardiomyopathies and other conditions. Patients were randomly selected in this study. Data were analyzed using 2-tailed Spearman's method. Our results demonstrated that in all conditions that we have studied, the velocity of RAA was compatible with that of LAA (agreement about 0.886).

Underlying heart diseases induce atrial dysfunction and subsequent increased risk of thromboembolic events. Most often, thrombotic materials arise in atrial appendages (1). As part of left atrium, left atrial appendage (LAA) is considered as a significant portion of cardiac anatomy. Thrombi are more frequently formed in LAA rather than RAA in patients with atrial fibrillation (AF); probably due to their different luminal surface area and size of neck (2). Thus, little attention has been paid to right atrial appendage (RAA) yet because of low clinical importance and its location. Indeed, ignorance rate for RAA screening in routine trans-esophageal echocardiography is high as RAA is a forgotten structure in the heart. But recently, RAA thrombosis and reduced function of RAA have been reported in AF cases compared with sinus rhythm state. The formed RAA thrombus in the setting of RAA dysfunction might be a potential source of pulmonary thromboembolism, thus the same attention put for LAA is needed for RAA (3). Positive correlation between LAA and RAA velocity was found in AF (4). The occurrence of RAA and LAA dysfunction has been observed in mitral stenosis and sinus rhythm due to increased atrial afterload and decreased atrial myocardial velocities $(5,6)$. But this correlation in other conditions is not completely evaluated. In this study, we aimed to evaluate the correlation between LAA and RAA velocities in different states. Thus, we have evaluated LAA and RAA velocities in 40 patients with different pathologies such as valvular and non-valvular AF, mitral stenosis, cardiomyopathies and other conditions. Patients were randomly selected in this study. Data were analyzed using 2-tailed Spearman's method. Our results demonstrated that in all of the conditions we have studied, the velocity of RAA was compatible with that of LAA (agreement about 0.886). This means that contractile function of LAA and RAA are compatible with each other. Based on these results, this correlation is independent to the studied state. Normal function and dysfunction of LAA is correlated to RAA, which means correlated atrial myocardial velocities and atrial afterloads. Dysfunction may be lower in one atrium which is associated with thrombus formation in the atrium with lower contraction. Decreased RAA velocity was seen in patients with AF and reduced LAA velocity. This correlation was seen to be associated with higher plasma concentration of brain natriuretic peptide (4). Variable blood flow patterns were seen in healthy subjects with increasing heart rate in both RAA and LAA by Mikael Kortz et al. (7). As shown by Bilge et al. (8), the correlation between echocardiography parameters of LAA and RAA was seen in cases with nonvalvular in contrast to valvular AF. Despite morphological 
differences between LAA and RAA, in this report we have shown positive correlation between RAA and LAA velocities in both disease and healthy states with high agreement rate. This inter appendage correlation was seen in both valvular and non-valvular AF cases, as well. This would suggest the presence of common pathologic remodeling for both appendages. Indeed, great attention to both atrial appendages should be paid in routine evaluation of atrial mechanical activity.

\section{Footnotes}

Authors' Contribution: Study concept and design: AA and MB. Analysis and interpretation of data: MB and SR. Drafting of the manuscript: AA and SR. Critical revision of the manuscript for important intellectual content: AA and MB. Statistical analysis: MB.

Conflict of Interests: No disclosure was received from the authors.

Funding/Support: None declared by the author.

\section{References}

1. Sahin T, Ural D, Kilic T, Bildirici U, Kozdag G, Agacdiken A, et al. Right atrial appendage function in different etiologies of permanent atrial fibrillation: a transesophageal echocardiography and tissue Doppler imaging study. Echocardiography. 2010;27(4):384-93. doi: 10.1111/j.15408175.2009.01027.x. [PubMed: 20331694].

2. Shinoda K, Hayashi S, Fukuoka D, Torii R, Watanabe T, Nakano T. Structural comparison between the right and left atrial appendages using multidetector computed tomography. Biomed Res Int. 2016;2016:6492183. doi: 10.1155/2016/6492183. [PubMed: 27900330]. [PubMed Central: PMC5120179].

3. Ozer O, Sari I, Davutoglu V. Right atrial appendage: forgotten part of the heart in atrial fibrillation. Clin Appl Thromb Hemost. 2010;16(2):21820. doi: 10.1177/1076029608323088. [PubMed: 18840628].

4. Kim BK, Heo JH, Lee JW, Kim HS, Choi BJ, Cha TJ. Correlation of right atrial appendage velocity with left atrial appendage velocity and brain natriuretic Peptide. J Cardiovasc Ultrasound. 2012;20(1):3741. doi: 10.4250/jcu.2012.20.1.37. [PubMed: 22509437]. [PubMed Central: PMC3324726].

5. Mahmoud KS, Al Kader MA. Comparison of left and right atrial appendages anatomy and function in patients with mitral stenosis and sinus rhythm. Egypt Heart J. 2012;64(4):219-25. doi: 10.1016/j.ehj.2012.07.004.

6. Cianciulli TF, Saccheri MC, Lax JA, Bermann AM, Mendez RJ, Guerra JE, et al. Right and left atrial appendage function in patients with mitral stenosis and sinus rhythm. Int J Cardiovasc Imaging. 2009;25(4):36370. doi: 10.1007/s10554-009-9430-9. [PubMed: 19184521].

7. Mikael Kortz RA, Delemarre BJ, van Dantzig JM, Bot H, Kamp O, Visser CA. Left atrial appendage blood flow determined by transesophageal echocardiography in healthy subjects. Am J Cardiol. 1993;71(11):976-81. doi: 10.1016/0002-9149(93)90917-2. [PubMed: 8465792].

8. Bilge M, Eryonucu B, Guler N, Erkoc R. Right atrial appendage function in patients with chronic nonvalvular atrial fibrillation.Jpn Heart J. 2000;41(4):451-62. doi: 10.1536/jhj.41.451. [PubMed: 11041096]. 